MATEC Web of Conferences 11,03010 (2014)

DOI: $10.1051 /$ matecconf $/ 20141103010$

(C) Owned by the authors, published by EDP Sciences, 2014

\title{
Comportement d'une Chaussée Souple sur Sol Support Gonflant
}

\author{
Adel Djellali. ${ }^{(1)}$, Abdelhafid Ounis ${ }^{(2)}$ Behrooz Saghafi ${ }^{(3)}$, Larbi Gadri ${ }^{(4)}$, Louafi messaoud ${ }^{(1)}$ and Ali Hamdane ${ }^{(1)}$ \\ ${ }^{1}$ Département de génie des Mines, Laboratoire LRMSM, Université Cheikh Larbi Tebessi, Tébessa, 12002 Algérie \\ ${ }^{2}$ Département de génie civil et hydraulique, Laboratoire LARGHYDE, Université Mohamed Khider, Biskra \\ ${ }^{3}$ Tarahan Parseh Transportation Research Institute, Street 55, Yousef Abad, Tehran 1436815361, Iran \\ ${ }^{4}$ Département de génie des Mines, Université Cheikh Larbi Tebessi, Tébessa, Tébessa, 12002 Algérie
}

\begin{abstract}
Résumé. Les déformations des sols fins sont la cause majeure des détériorations des chaussées souples. Elles sont en relation avec les conditions climatiques qui favorisent la variation de l'état hydrique (fluctuation de la teneur en eau) accentués par une augmentation ou diminution du volume de sol. Cet article présente une étude géotechnique spécifique complète, il détermine la cause principale des dégradations des chaussées souples sur sols gonflants, par indication que le gonflement est le générateur de ces dégâts. Les données ont été traitées par une analyse statistique en composante principale ACP. Le code de calcul Plaxis à été utilité pour vérifier les déplacements verticaux du corps de chaussée et d'étudier le comportement de ces sols vis-àvis au remblai et du corps de la chaussée.
\end{abstract}

\section{Introduction}

Les déformations des sols fins sont la cause majeure de nombreux dommages des structures et ouvrages du génie civil. Elles sont en relation avec les conditions climatiques qui favorisent la variation de l'état hydrique dans le sol accentuée par une augmentation ou diminution du volume de sol causée par la fluctuation de la teneur en eau [1]. Dans le cas des remblais routier ces dégâts sont considérables soit en matière de construction « duré de vie très limitée », soit sur le coût d'entretient. Ceci se manifestent par plusieurs déformations (fissures longitudinales ou transversales, nid de poule, etc....), dans la chaussée surtout en phase de construction en période sèche où dans une région soumise à de grandes périodes de sècheresse. Par conséquent, la ville de Tébessa-Algérie- est formée généralement et en grande partie d'argiles soit limoneuses ou marneuses. La région connait également de grandes périodes sèches en été ou durant toute l'année avec des précipitations soudaines courtes et torrentielles. Ces sols qui ont un caractère gonflant, sont l'assise de la majorité des réseaux routiers de la ville (RN10, RN16 et RN 82) qui subissent de larges déformations et dégradations. Cet article s'intéresse particulièrement à la précision de la cause originale de ces dégradations en indiquant que le gonflement est la cause principale de ces dégâts par une étude géotechnique spécifique et complète. Les données seront traitées par une analyse statistique en composante principale ACP. Nous étudierons par la suite le comportement de ces sols vis-àvis du remblai et la chaussée par un modèle numérique.

\section{Localisation de la zone d'étude}

Une chaussée souple est construite sur un sol support gonflant dans la ville de Tébessa en Algérie. La ville se trouve dans un bassin d'effondrement entourée de montagnes avec une altitude moyenne de $800 \mathrm{~m}$. Elle est bordée au nord par la ville de Souk-Ahras, au sud par El'Oued, à l'Est par la frontière tunisienne et à l'ouest par la ville de Khenchella. La route commence à l'intersection des RN10 et RN 82 (route d'El Kouif) et s'achève à l'intersection de la RN16 (route d'El Malabiod) et route de Bekkaria (Fig. 1).

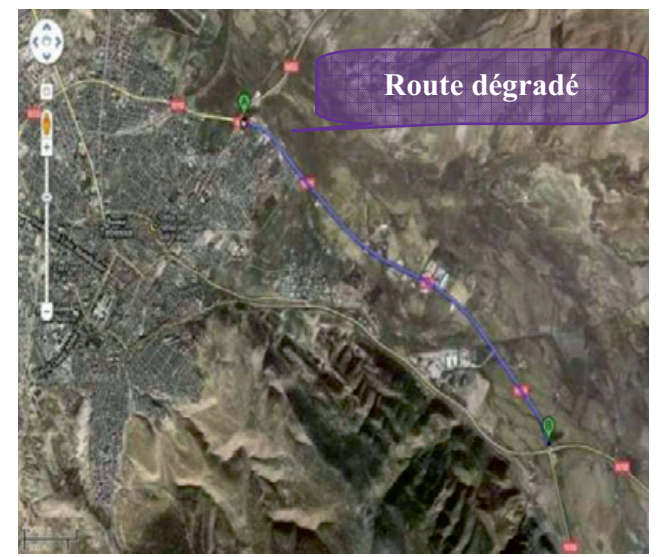

Fig.1. Situation géographique de la zone d'étude [2]. 


\section{Classification des sols}

Dans le cadre de la réhabilitation de la route nationale RN 10, qui a subi d'énormes dégradations dans la structure de la chaussée (Fig. 1), le programme d'analyse du sol a été mis en place par 10 sondages carottés à $8 \mathrm{~m}$ de profondeur et 21 puits de 2 à $3 \mathrm{~m}$ de profondeur. Afin d'établir le profil géologique du site et d'avoir suffisamment d'échantillons pour essais au laboratoire. Les données ont été triées et sélectionnés afin de faciliter leur exploitation. Ils sont répartis sur une longueur de $5 \mathrm{Kms}$. Analyses visuelles des échantillons a montré que nous sommes en présence d'argiles marneuses, argile limoneuse et marneuse. La classification selon le diagramme de Casagrande qui apprécier non seulement la plasticité et la nature des sols, mais a également fourni un aperçu sur leur potentiel de gonflement, montrent que les sols sont des argiles inorganiques de moyenne à haute plasticité. La classification des Dakshanamurty et Raman (1973) qui est basée sur les mêmes paramètres de classification de Casagrande [3], montre que le potentiel de gonflement est moyenne à élevée.

\subsection{Classification par des cercles de corrélation}

L'étude de la corrélation linéaire entre les propriétés géotechniques et les paramètres de gonflement du sol support a été faite sur la base des résultats obtenus dans un programme de laboratoire expérimental complet. L'objectif principal de ce programme est de contribuer à améliorer les connaissances sur le comportement des chaussées souples sur sols gonflants. Le traitement statistique par composantes principales (ACP) permet de sélectionner des échantillons représentatifs des populations recueillies [4]. L'analyse des plans F1F2, permet de distinguer plusieurs groupes de variables: l'une est près de l'axe F1 et les autres près de l'axe F2. Plus précisément, on retrouve deux groupes qui sont respectivement :

- $\quad$ LL, PL, W, $\gamma_{\mathrm{h}}$, Ps and Cs groups ;
- $\quad \gamma_{\mathrm{d}}, \mathrm{CaCO} 3, \mathrm{BM}$ and CBR groups.

ous pouvons observer que le premier groupe, c'est à dire, LL, PL, W, $\gamma_{h}$, Ps et Cs qui sont corrélée négativement par rapport à $F 1$, ce qui indiquent que ces paramètres en particulier la teneur en eau $\mathrm{W} \%$ influent directement et négativement sur le comportement des sols gonflants. Dans l'autre côté le groupe de BM, CBR, $\gamma \mathrm{d}$ et $\mathrm{CaCO} 3$ sont corrélée positivement par rapport $\mathrm{F} 1$, donc ils influencent positivement sur le comportement des sols gonflants exceptionnellement la valeur de bleu de méthylène.

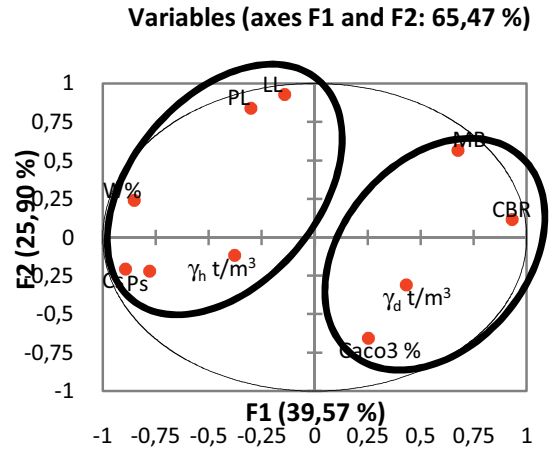

Fig. 2.Cercles des corrélations dans le repère F1F2.

\section{Modélisation}

Le modèle est une structure de chaussée souple existante qui a subi de multiples dégradations après une année de sa mise en œuvre (ville de TébessaAlgérie). Cette route est construite sur un sol support de type argiles limoneuses brunâtres, l'épaisseur totale de la chaussée est de $760 \mathrm{~mm}$, dont $200 \mathrm{~mm}$ couche de forme en tuf calcaire, $200 \mathrm{~mm}$ couche de fondation en grave concassée, $200 \mathrm{~mm}$ couche base en grave concassé et la couche de roulement en béton bitumineux de $60 \mathrm{~mm}$ d'épaisseur (Fig.3). Cette structure a subi des déformations, notamment: des fissures transversales moyennes, fissures en carrelage moyenne, avec ornière à grand rayon de l'ordre de $90 \times 10^{-3} \mathrm{~m}$ de profondeur et apparition des fissures dans les accotements. L'essai œdométrique à gonflement libre a révélé que la déformation verticale (soulèvement) a une valeur maximale de 2,06 mm avec une pression de gonflement de $350 \mathrm{kN} / \mathrm{m} 2$.

Tableau 1. Paramètres d'entrée du modèle.

\begin{tabular}{c|cccc}
\hline Matériaux & $\begin{array}{c}\text { Couche } \\
\text { de } \\
\text { roulement }\end{array}$ & $\begin{array}{c}\text { Couche de } \\
\text { base } \\
\text { grave } \\
\text { concassée }\end{array}$ & $\begin{array}{c}\text { Couche de } \\
\text { fondation } \\
\text { grave } \\
\text { concassée }\end{array}$ & $\begin{array}{c}\text { Couche } \\
\text { de } \\
\text { forme } \\
\text { Tuf }\end{array}$ \\
\hline $\begin{array}{c}\text { Épaisseur } \\
\text { mm } \\
\text { Module de }\end{array}$ & 60 & 200 & 200 & 200 \\
$\begin{array}{c}\text { Young, MPa } \\
\text { Coefficient } \\
\text { de poisson } \\
\text { densité, } \\
\text { Kn/m }\end{array}$ & 0,35 & 0,35 & 0,35 & 0,35 \\
$\begin{array}{c}\text { Cohésion, } \\
\text { KPa }\end{array}$ & 25 & 21,2 & 22,0 & 20 \\
$\begin{array}{c}\text { Angle de } \\
\text { frottement }\end{array}$ & -- & 30 & 20 & 90 \\
\hline
\end{tabular}




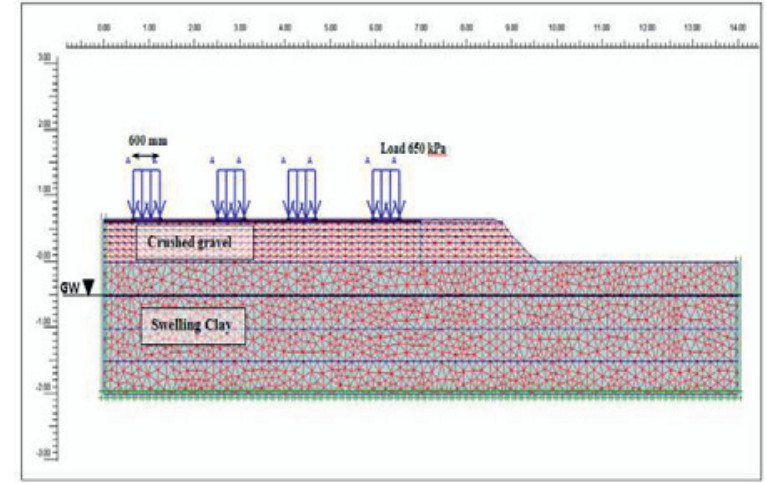

Fig. 3. Modèle numérique : discrétisation et conditions aux limites.

Le critère de rupture statique où il est habituellement adapté en géotechnique, et avec les chaussées est le critère de rupture de Mohr-Coulomb qui est le mieux accoutumé. Le comportement du remblai relie le rapport de rupture au rapport des contraintes déviatoriques, dans ce cas le rapport de rupture $\mathrm{R}$ peut s'écrire de la façon suivante :

$$
\begin{gathered}
R=\frac{q}{q_{0}+M p^{\prime}} \\
M=\frac{6 \cdot \sin \emptyset}{3-\sin \varnothing} \\
q=\frac{c \cdot 6 \cdot \cos \emptyset}{3-\sin \emptyset}
\end{gathered}
$$

D'où $R$ est le rapport de rupture

$\mathrm{q} \quad$ contrainte déviatorique, $\mathrm{kPa}$

$\mathrm{q}_{0} \quad$ contrainte déviatorique, quand $\mathrm{q}=0$

C cohésion, $\mathrm{kPa}$

M la pente de la ligne de rupture dans la plan $\mathrm{p}$ - $\mathrm{q}$

p' pression hydrostatique, $\mathrm{kPa}$

$\varnothing \quad$ angle de frottement interne.

Puisque le sol support a un caractère un gonflent et est complètement saturé, il a été modélisé par le modèle des sols mou, ce modèle prend en compte les paramètres suivants : les contraintes dépendent de la rigidité du sol (le comportement de compression logarithmique); la distinction entre le chargement primaire et le déchargement-rechargement (compatible avec le comportement de gonflement) ; prendre en mémoire la contrainte de près-consolidation [5]. Le modèle des sols mous suppose qu'il y a une relation logarithmique entre la déformation volumétrique $\mathcal{E}_{v}$, et la contrainte effective $p$ 'sous la relation :

$\varepsilon_{\mathrm{V}}-\varepsilon_{\mathrm{V}}^{0}=-\lambda^{*} \ln \left(\frac{p^{\prime \prime}}{p^{0}}\right)$

Avec $\lambda^{*}$ est l'indice de compression modifié.

$\mathrm{Au}$ cours de déchargement-rechargement isotropique de différents cheminements de contraintes se tracent, où elle peut se formuler comme suit :

$\varepsilon_{\mathrm{v}}^{\mathrm{e}}-\varepsilon_{\mathrm{v}}^{\mathrm{e} 0}=-\kappa^{*} \ln \left(\frac{p^{\prime \prime}}{p^{0}}\right)$

Avec $\lambda^{*}$ est l'indice de gonflement modifié. [6].

\section{Analyse des résultats}

La figure 4 indique clairement que les déplacements se concentrent en grande partie sous la zone de transition entre chaussée-accotement dont le sol support- corps de la chaussée et l'accotement- avec une déformation de l'ordre de $80,89 \times 10^{-3}$ ce qui elle est proche avec celle mesuré sur le terrain $(90 \mathrm{x}$ $10^{-3}$ ), par contre dans la partie environnante de la chaussée le sol support est faiblement dérangé

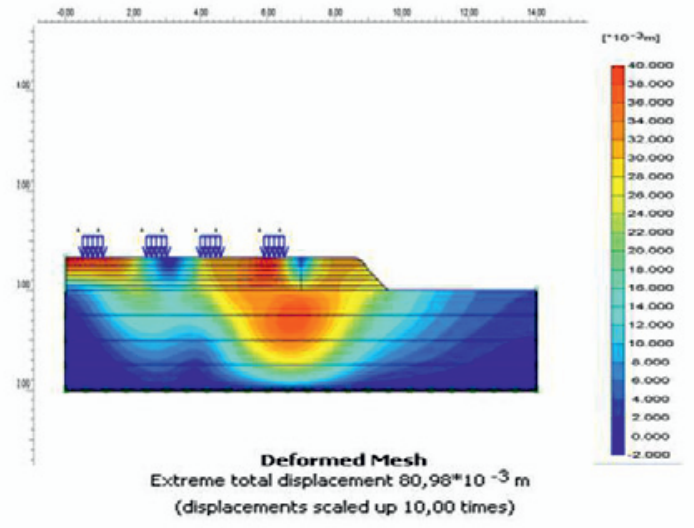

Fig. 4. Déformations totales de la structure.

L'examen des courbes de la figure 5, qui représente le cheminement des contraintes pour le sol support, montre que le sol support est de nature argile gonflante a un comportement élasto-plastique avec écrouissage.

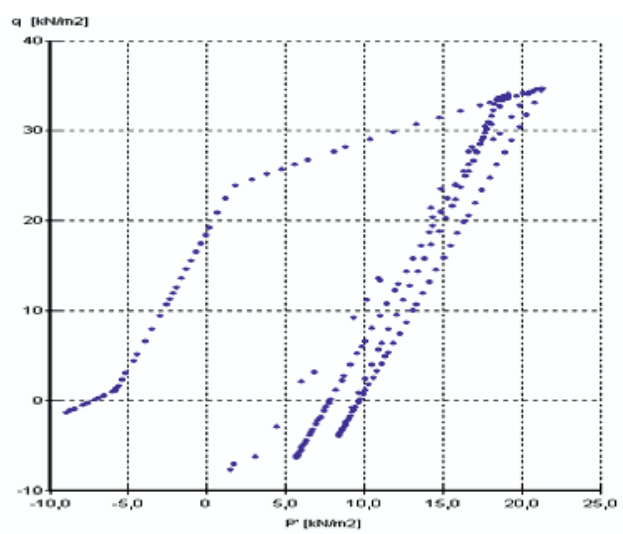

Fig. 5. Cheminements des contraintes

\section{Conclusion}

Cette étude est porté sur la modélisation d'une chaussée souple sur sols gonflants montre que :

- La distribution des contraintes dans le corps du remblai et la structure de la chaussée peut être 
réellement modélisée par le programme d'éléments finis Plaxis ;

- Le choix sur modèle de comportement du sol affecte sévèrement les distributions des contraintes, et il s'étende jusqu'aux déformations résilientes ;

- Dans le calcul des déformations permanentes des chaussées sur sols gonflants, il est important de modéliser la distribution des contraintes avec un modèle conventionnelle qui donne une distribution de contraintes le plus proche de réalité d'où le modèle des sols mous pour le sol support, et le modèle de Mohr-Coulomb pour le corps de la chaussée ;

- La combinaison du modèle Mohr-Coulomb dans le corps de chaussée et le modèle des sols mous pour le sol support donne une bonne distribution des contraintes en comparent ces résultats à la réalité (Fissures sur la chaussée le sur 1'accotement);

- Le sol support à un comportement élastoplastique avec écrouissage ;

- La source réelle des déformations qui se manifeste sur le corps de chaussée, est non seulement l'intensité du trafic mais, c'est la résultante des pressions de gonflement induite par le sol support et la charge du trafic.

\section{Références}

1. D.R. Snethen, F.C. Townsend, L.D.Johnson, D. M. Patrick, P. J. Vedros. "Review of Engineering Experiences with Expansive soils in Highway Subgrades". US Army Engineer Water Ways Experiment Station, FHWA, 009751, USA (1975)

2. Available online: $<$ http://maps.google.fr. $>$.

3. V. Dakshanamanthy, V. Raman. A simple method of identifying an expansive soil. Soils and Foundations, Japanese Society of Soil Mechanics and Foundation Engineering 13 (1), 97-104 (1973)

4. A.Sridharan, Y.Gurtug. Swelling behaviour of compacted fine-grained soils. Elsevier Scientific Publishing, Engineering Geology 72, 9-18 (2004)

5. R.B.J. Brinkgreve. Plaxis 2D, Version 8 manual, Material models, Delft, Balkema (2002)

6. R.B.J. Brinkgreve. Plaxis 2D, Version 8 manual, Reference Manual, Delft University of Technology \& PLAXIS bv, Pays-Bas, pp 50$162(2003)$ 